\title{
An Improvement of a Non-uniform Concentration Inequality for Randomized Orthogonal Array Sampling Designs
}

\author{
Kittipong Laipaporn (Corresponding author) \\ Department of Mathematics, School of Science \\ Walailak University, Nakhon Si Thammarat 80160, Thailand \\ E-mail: lkittipo@wu.ac.th \\ Kritsada Sungkamongkol \\ Department of Mathematics, Faculty of Science \\ Chulalongkorn University, Bangkok 10330, Thailand \\ E-mail: k_man2002@hotmail.com
}

\begin{abstract}
Let $f$ be an integrable function from $\mathbb{R}^{3}$ to $\mathbb{R}$ and $\mu=\int_{[0,1]^{3}} f(x) d x$. A simple estimator of $\mu$ is $\hat{\mu}=\frac{1}{n} \sum_{i=1}^{n} f \circ X_{i}$ where $X_{1}, X_{2}, \ldots X_{n}$ are independent random vectors and uniformly distributed on $[0,1]^{3}$. In 2006, Neammanee and Laipaporn used the orthogonal array to choose the points $X_{i}$ 's and established a non-uniform concentration inequality. In this article, we improve their result.
\end{abstract}

Keywords: Computer experiment, Orthogonal array, Non-uniform concentration inequality

\section{Introduction}

Let $f:[0,1]^{k} \rightarrow \mathbb{R}$ be a measurable function and let $X$ be a uniform random vector on $[0,1]^{k}$. In computer experiments, for examples, the behavior of nuclear weapon, climate change, the electrical circuit and the fluid flow problems, we simply refer the collection of input as $x$, the simulator of each circumstance as $f$, and the output of experiment as $f(x)$. The expected value,

$$
\mu=E(f \circ X)=\int_{[0,1]^{k}} f(x) d x
$$

is cosidered as the representative of output tendency. Sometimes the function $f$ is not easily computed or the dimension $k$ is very high. It is widely acceptable that Monte Carlo method is competitive for high-dimensional problem ((Davis, P. J.,1984) and (Evans, M., 2000)). That is, we randomly choose $n$ points from $[0,1]^{k}$, say $X_{1}, X_{2}, \ldots X_{n}$, and define

$$
\hat{\mu}=\frac{1}{n} \sum_{i=1}^{n} f \circ X_{i}
$$

to estimate the expected value $E(f \circ X)$.

Alternatively, the selection of the $n$ points $X_{1}, X_{2}, \ldots, X_{n}$ are presented by many mathematicians such as lattice point sampling (Patterson, H. D., 1954), Latin hypercube sampling (Owen, A. B., 1992a), orthogonal array sampling (Owen, A. B., 1992b) and scrambled net sampling (Owen, A. B., 1997) and (Tang, B., 1993). In this article, we use the orthogonal array sampling which provide many applications ((Davis, J., 2007), (Lazic, L., 2008), (Lee, K. H., 2003) and (Lin, K. P., 2008)) and the definition is stated as follow.

Let $d, n$ and $t$ be positive integers with $t \leq d$. An orthogonal array of strength $t$ is a matrix of $n$ rows and $d$ columns with elements taken from the set $\{0,1, \ldots, q-1\}$ such that in any $n \times t$ submatrix, each of the $q^{t}$ possible rows occurs the same number of times. The class of all such arrays is denoted by $O A(n, d, q, t)$. In 1996, Loh considered the function $f:[0,1]^{3} \rightarrow \mathbb{R}$ and used $A=\left(a_{i, j}\right) \in O A\left(q^{2}, 3, q, 2\right)$ to approximate $\mu$ by the estimator 


$$
\hat{\mu}=\frac{1}{q^{2}} \sum_{i=1}^{q^{2}} f \circ X\left(\pi_{1}\left(a_{i, 1}\right), \pi_{2}\left(a_{i, 2}\right), \pi_{3}\left(a_{i, 3}\right)\right)
$$

where

(a) $\pi_{1}, \pi_{2}, \pi_{3}$ are random permutations of $\{0,1, \ldots, q-1\}$,

(b) for any $i_{1}, i_{2}, i_{3} \in\{0,1, \ldots, q-1\}$ and $j \in\{1,2,3\}$

$$
\begin{aligned}
& X\left(i_{1}, i_{2}, i_{3}\right)=\left(X_{1}\left(i_{1}, i_{2}, i_{3}\right), X_{2}\left(i_{1}, i_{2}, i_{3}\right), X_{3}\left(i_{1}, i_{2}, i_{3}\right)\right), \\
& X_{j}\left(i_{1}, i_{2}, i_{3}\right)=\frac{i_{j}+U_{i_{1}, i_{2}, i_{3}, j}}{q} \text { and }
\end{aligned}
$$

$U_{i_{1}, i_{2}, i_{3}, j}$ 's are uniform random variables on $[0,1]$ and

(c) $U_{i_{1}, i_{2}, i_{3}, j}$ 's and $\pi_{k}$ 's are all stochastically independent.

In 1996, Loh was the first who considered the normal approximation of

$$
W=\frac{\hat{\mu}-\mu}{\sqrt{\operatorname{Var}(\hat{\mu})}}
$$

where $\operatorname{Var}(\hat{\mu})>0$. He gave a uniform bound of orthogonal array sampling designs and Laipaporn and Neammanee improved the bound to the rate $O\left(q^{-\frac{1}{2}}\right)$ in (Laipaporn, K., 2008). Besides the normal approximation, they also investigated a non-uniform concentration inequality of $W$ in Theorem 1(Laipaporn, K., 2006).

Theorem 1 Assume that $E(f \circ X)^{4}<\infty$. Then, there exists a constant $\mathrm{C}$ such that

$$
P(z \leq W \leq w) \leq \frac{C}{1+z}(w-z)+\frac{1}{1+z} O\left(\frac{1}{\sqrt{q}}\right) \text {, as } q \rightarrow \infty,
$$

for any real number $0<z \leq w$.

In this paper we will improve the bound of Theorem 1 as in Theorem 2.

Theorem 2 Assume that $E(f \circ X)^{4}<\infty$. Then, there exists a constant $\mathrm{C}$ such that

$$
P(z \leq W \leq w) \leq \frac{C}{(1+z)^{3}}(w-z)+\frac{1}{(1+z)^{2}} O\left(\frac{1}{\sqrt{q}}\right), \text { as } q \rightarrow \infty,
$$

for any real number $0<z \leq w$.

\section{Auxiliary Results}

To prove Theorem 2, we need the following lemmas and some notations.

In 1996, Loh introduced a random function $\rho_{\pi}:\{0,1, \ldots, q-1\}^{2} \rightarrow\{0,1, \ldots, q-1\}$

which is defined by

$$
\left(i_{1}, i_{2}, \rho_{\pi}\left(i_{1}, i_{2}\right)\right)=\left(\pi_{1}\left(a_{i, 1}\right), \pi_{2}\left(a_{i, 2}\right), \pi_{3}\left(a_{i, 3}\right)\right) \text { for some } i \in\left\{1,2, . ., q^{2}\right\}
$$

For each $i, j$ and $k \in\{0,1, \ldots, q-1\}$, and $z>0$, we let $I$ and $K$ be uniformly distributed random variables on $\{0,1, \ldots, q-1\}$, $(I, K)$ uniformly distributed on $\{(i, k) \mid i, k=0,1, \ldots, q-1, i \neq k\}$.

Let

$$
\begin{aligned}
& Y_{z}(i, j, k)=Y(i, j, k) \mathbb{I}(|Y(i, j, k)|>1+z), \\
& \widehat{Y}_{z}(i, j, k)=Y(i, j, k) \mathbb{I}(|Y(i, j, k)| \leq 1+z), \\
& \widehat{Y}=\sum_{i=0}^{q-1} \sum_{j=0}^{q-1} \widehat{Y}_{z}\left(i, j, \rho_{\pi}(i, j)\right) \\
& \widetilde{Y}=\widehat{Y}-\widehat{S}_{1, z}-\widehat{S}_{2, z}+\widehat{S}_{3, z}+\widehat{S}_{4, z} \\
& \text { where } \quad \widehat{S}_{1, z}=\sum_{j=0}^{q-1} \widehat{Y}_{z}\left(I, j, \rho_{\pi}(I, j)\right), \quad \widehat{S}_{2, z}=\sum_{j=0}^{q-1} \widehat{Y}_{z}\left(K, j, \rho_{\pi}(K, j)\right) \text {, } \\
& \widehat{S}_{3, z}=\sum_{j=0}^{q-1} \widehat{Y}_{z}\left(I, j, \rho_{\pi}(K, j)\right), \quad \widehat{S}_{4, z}=\sum_{j=0}^{q-1} \widehat{Y}_{z}\left(K, j, \rho_{\pi}(I, j)\right) .
\end{aligned}
$$

and 
Lemma 3 ((Laipaporn, K., 2006) and (Loh, W.L., 1996)) Assume that $E(f \circ X)^{r}<\infty$ for some positive even integer $r$. Then

1. $E|\widetilde{Y}-\widehat{Y}|^{r}=O\left(\frac{1}{q^{\frac{r}{2}}}\right)$, as $q \rightarrow \infty$,

2. $\sum_{i=0}^{q-1} \sum_{j=0}^{q-1} \sum_{k=0}^{q-1} E Y^{r}(i, j, k)=O\left(q^{3-r}\right)$, as $q \rightarrow \infty$,

3. For any positive integer $n$ and $t$ such that $n+t$ is an even number and $n+t \leq r$,

$$
\sum_{i=0}^{q-1} \sum_{j=0}^{q-1} \sum_{k=0}^{q-1} E\left|Y_{z}^{n}(i, j, k)\right|=\frac{O\left(q^{3-n-t}\right)}{(1+z)^{t}} \text {, as } q \rightarrow \infty .
$$

Lemma 4 (Laipaporn, K., 2006) Let $g: \mathbb{R} \rightarrow \mathbb{R}$ be a continuous and piecewise continuously differentiable function, then

$$
E \widehat{Y} g(\widehat{Y})=E \int_{-\infty}^{\infty} g^{\prime}(\widehat{Y}+t) K(t) d t+\widetilde{\Delta} g(\widehat{Y})
$$

where

$$
K(t)=\frac{q-1}{4}(\widetilde{Y}-Y)(\mathbb{I}(0 \leq t \leq \widetilde{Y}-Y)-\mathbb{I}(\widetilde{Y}-Y \leq t<0))
$$

and

$$
\widetilde{\Delta} g(\widehat{Y})=\frac{1}{q} \operatorname{Eg}(\widehat{Y}) \sum_{i=0}^{q-1} \sum_{j=0}^{q-1} \sum_{k=0}^{q-1} \widehat{Y}_{z}(i, j, k)
$$

Lemma 5 (Laipaporn, $K$., 2006) If $E(f \circ X)^{2}<\infty$, then $E(\widehat{Y}-\widetilde{Y})^{2}=\frac{4}{q}+O\left(\frac{1}{q^{2}}\right)$, as $q \rightarrow \infty$.

Lemma 6 (Laipaporn, $K ., 2007)$ If $E(f \circ X)^{4}<\infty$, then
1. $E\left(\sum_{i=0}^{q-1} \sum_{j=0}^{q-1} \sum_{k=0}^{q-1} Y(i, j, k)\right)^{4} \leq O\left(q^{2}\right)$, as $q \rightarrow \infty$
2. $E\left(\sum_{i=0}^{q-1} \sum_{j=0}^{q-1} \sum_{k=0}^{q-1} Y_{z}(i, j, k)\right)^{4} \leq \frac{1}{(1+z)^{2}} O\left(\frac{1}{q^{2}}\right)$, as $q \rightarrow \infty$.

Lemma 7 Assume that $E(f \circ X)^{4}<\infty$. Let $\gamma=\max \left(\frac{q(q-1)}{4(q-4)} E|\widehat{Y}-\widetilde{Y}|^{3}, \frac{1}{\sqrt{q}}\right)$ and

$$
\begin{gathered}
U_{\gamma}=\sum_{i \neq k}\left|\sum_{j=0}^{q-1}\left\{\widehat{Y}_{z}\left(i, j, \rho_{\pi}(i, j)\right)+\widehat{Y}_{z}\left(k, j, \rho_{\pi}(k, j)\right)-\widehat{Y}_{z}\left(i, j, \rho_{\pi}(k, j)\right)-\widehat{Y}_{z}\left(k, j, \rho_{\pi}(i, j)\right)\right\}\right| \\
\times \min \left(\gamma, \sum_{i \neq k}\left|\sum_{j=0}^{q-1}\left\{\widehat{Y}_{z}\left(i, j, \rho_{\pi}(i, j)\right)+\widehat{Y}_{z}\left(k, j, \rho_{\pi}(k, j)\right)-\widehat{Y}_{z}\left(i, j, \rho_{\pi}(k, j)\right)-\widehat{Y}_{z}\left(k, j, \rho_{\pi}(i, j)\right)\right\}\right|\right) .
\end{gathered}
$$

Then

1. $E U_{\gamma} \geq 3 q+O(1)$, as $q \rightarrow \infty$,

2. $\operatorname{Var}\left(U_{\gamma}\right) \leq \gamma^{2} O\left(q^{2}\right)$.

Proof. (1.) From the fact that $\min (a, b) \geq b-\frac{b^{2}}{4 a}$ for any $a, b>0$, 


$$
\begin{aligned}
E\left(U_{\gamma}\right) & =q(q-1) E(\widehat{Y}-\widetilde{Y}) \min (\gamma,|\widehat{Y}-\widetilde{Y}|) \\
& \geq q(q-1) E(\widehat{Y}-\widetilde{Y})^{2}-\frac{q(q-1)}{4 \gamma} E|\widehat{Y}-\widetilde{Y}|^{3} \\
& \geq q(q-1) E(\widehat{Y}-\widetilde{Y})^{2}-\frac{q(q-1)}{4\left(\frac{q(q-1)}{4(q-4)} E|\widehat{Y}-\widetilde{Y}|^{3}\right)} E|\widehat{Y}-\widetilde{Y}|^{3} .
\end{aligned}
$$

By Lemma 5, we have

$$
\begin{aligned}
E\left(U_{\gamma}\right) & \geq q(q-1)\left(\frac{4}{q}+O\left(\frac{1}{q^{2}}\right)\right)-(q-4) \\
& =3 q+O(1)
\end{aligned}
$$

(2.) By using the same argument of Lemma 2.5(Laipaporn, K., 2006), we have

$$
\operatorname{Var}\left(U_{\gamma}\right) \leq \frac{q(q-1)}{4}\left\{\gamma^{2} O\left(\frac{1}{q}\right)+\frac{\gamma^{2}}{(1+z)^{2}} O\left(\frac{1}{q^{2}}\right)\right\}+\frac{1}{q(q-1)}\left\{\gamma^{2}\left(q^{4}\right)\right\}=\gamma^{2} O\left(q^{2}\right) .
$$

Lemma 8. If $E(f \circ X)^{4}<\infty$, then $E \widehat{Y}^{4}=O(1)$, as $q \rightarrow \infty$.

Proof. First we note that

$$
\begin{aligned}
E \widehat{Y}^{4} & =E\left(\sum_{i, j} \widehat{Y}_{z}\left(i, j, \rho_{\pi}(i, j)\right)\right)^{4} \\
& =E\left(\sum_{i, j} Y\left(i, j, \rho_{\pi}(i, j)\right)-\sum_{i, j} Y_{z}\left(i, j, \rho_{\pi}(i, j)\right)\right)^{4} \\
& \leq C E W^{4}+C E\left(\sum_{i, j} Y_{z}\left(i, j, \rho_{\pi}(i, j)\right)\right)^{4} .
\end{aligned}
$$

In (Laipaporn, K., 2009), we proved that $E W^{4}=O(1)$, so it remains to show that $E\left(\sum_{i, j} Y_{z}\left(i, j, \rho_{\pi}(i, j)\right)\right)^{4}=O(1)$.

Note that $\quad E\left(\sum_{i, j} Y_{z}\left(i, j, \rho_{\pi}(i, j)\right)\right)^{4}=B_{1}+B_{2}+B_{3}+B_{4}+B_{5}$

where

$$
\begin{aligned}
& B_{1}=\sum_{i, j} E Y_{z}^{4}\left(i, j, \rho_{\pi}(i, j)\right) \\
& B_{2}=\sum_{i_{1}, j_{1}} \sum_{\substack{i_{2}, j_{2} \\
\left(i_{2}, j_{2}\right) \neq\left(i_{1}, j_{1}\right)}} E Y_{z}^{3}\left(i_{1}, j_{1}, \rho_{\pi}\left(i_{1}, j_{1}\right)\right) Y_{z}\left(i_{2}, j_{2}, \rho_{\pi}\left(i_{2}, j_{2}\right)\right) \\
& B_{3}=\sum_{i_{1}, j_{1}} \sum_{\substack{i_{2}, j_{2} \\
\left(i_{2}, j_{2}\right) \neq\left(i_{1}, j_{1}\right)}} E Y_{z}^{2}\left(i_{1}, j_{1}, \rho_{\pi}\left(i_{1}, j_{1}\right)\right) Y_{z}^{2}\left(i_{2}, j_{2}, \rho_{\pi}\left(i_{2}, j_{2}\right)\right)
\end{aligned}
$$

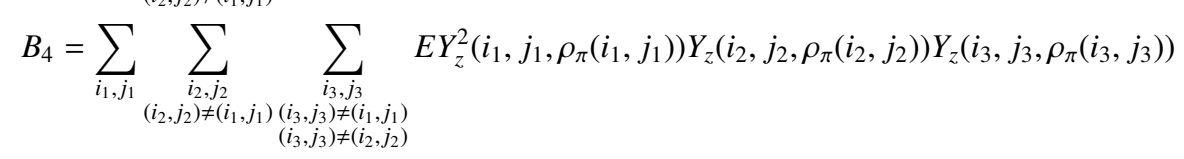

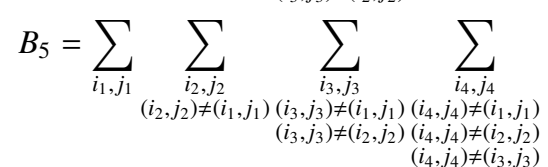

$$
\begin{aligned}
& E Y_{z}\left(i_{1}, j_{1}, \rho_{\pi}\left(i_{1}, j_{1}\right)\right) Y_{z}\left(i_{2}, j_{2}, \rho_{\pi}\left(i_{2}, j_{2}\right)\right) Y_{z}\left(i_{3}, j_{3}, \rho_{\pi}\left(i_{3}, j_{3}\right)\right) Y_{z}\left(i_{4}, j_{4}, \rho_{\pi}\left(i_{4}, j_{4}\right)\right) .
\end{aligned}
$$


By Lemma 3(3) we have

$$
\begin{aligned}
& \left|B_{1}\right| \leq \frac{1}{q} \sum_{i, j, k} E\left|Y_{z}^{4}(i, j, k)\right|=\frac{1}{q} O\left(q^{3-4}\right)=O\left(\frac{1}{q^{2}}\right), \\
& \left|B_{2}\right| \leq \frac{1}{q^{2}}\left[\sum_{i_{1}, j_{1}, k_{1}} E\left|Y_{z}^{3}\left(i_{1}, j_{1}, k_{1}\right)\right|\right]\left[\sum_{i_{2}, j_{2}, k_{2}} E\left|Y_{z}\left(i_{2}, j_{2}, k_{2}\right)\right|\right] \\
& \left.=\frac{1}{q^{2}(1+z)} O\left(q^{3-3-1}\right)\left(\frac{1}{1+z}\right) O^{(} q^{3-1-1}\right) \\
& =\frac{1}{(1+z)^{2}} O\left(\frac{1}{q^{2}}\right) \\
& \left|B_{3}\right| \leq \frac{1}{q^{2}}\left[\sum_{i, j, k} E\left|Y_{z}^{2}(i, j, k)\right|\right]^{2} \\
& =\frac{1}{q^{2}}\left(\frac{1}{(1+z)^{2}} O\left(q^{3-2-2}\right)\right)^{2} \\
& =\frac{1}{(1+z)^{4}} O\left(\frac{1}{q^{4}}\right) \text {, } \\
& \left|B_{4}\right| \leq \frac{1}{q^{3}}\left[\sum_{i_{1}, j_{1}, k_{1}} E\left|Y_{z}^{2}\left(i_{1}, j_{1}, k_{1}\right)\right|\right]\left[\sum_{i_{2}, j_{2}, k_{2}} E\left|Y_{z}\left(i_{2}, j_{2}, k_{2}\right)\right|\right]^{2} \\
& =\frac{1}{q^{3}(1+z)^{2}} O\left(q^{3-2-2}\right)\left(\frac{1}{(1+z)} O\left(q^{3-1-1}\right)\right)^{2} \\
& =\frac{1}{(1+z)^{4}} O\left(\frac{1}{q^{2}}\right) \\
& \text { and }\left|B_{5}\right| \leq \frac{1}{q^{4}}\left[\sum_{i, j, k} E\left|Y_{z}(i, j, k)\right|\right]^{4} \\
& =\left(\frac{1}{q^{4}}\right)\left(\frac{1}{(1+z)} O\left(q^{3-1-1}\right)\right)^{4} \\
& =\frac{1}{(1+z)^{4}} O(1) \text {. }
\end{aligned}
$$

Hence $E\left(\sum_{i, j} Y_{z}\left(i, j, \rho_{\pi}(i, j)\right)\right)^{4}=O(1)$. Therefore $E \widehat{Y}^{4}=O(1)$.

\section{Proof of Theorem 2}

Proof. Note that $P(z \leq W \leq w) \leq P(W \neq \widehat{Y})+P(z \leq \widehat{Y} \leq w)$ and by Lemma 3(2),

$$
\begin{aligned}
P(W \neq \widehat{Y}) & =P\left(\sum_{i=0}^{q-1} \sum_{j=0}^{q-1} \mathbb{I}\left(\left|Y\left(i, j, \rho_{\pi}(i, j)\right)\right|>1+z\right) \geq 1\right) \\
& \leq E\left(\sum_{i=0}^{q-1} \sum_{j=0}^{q-1} \mathbb{I}\left(\left|Y\left(i, j, \rho_{\pi}(i, j)\right)\right|>1+z\right)\right) \\
& =\frac{1}{q} \sum_{i=0}^{q-1} \sum_{j=0}^{q-1} \sum_{k=0}^{q-1} E \mathbb{I}(|Y(i, j, k)|>1+z) \\
& \leq \frac{1}{q} \sum_{i=0}^{q-1} \sum_{j=0}^{q-1} \sum_{k=0}^{q-1} \frac{E|Y(i, j, k)|^{4}}{(1+z)^{4}} \\
& \leq \frac{1}{q(1+z)^{4}} O\left(q^{3-4}\right) \\
& =\frac{1}{(1+z)^{4}} O\left(\frac{1}{q^{2}}\right) .
\end{aligned}
$$


If we can show that $P(z \leq \widehat{Y} \leq w) \leq \frac{C}{(1+z)^{3}}(w-z)+\frac{1}{(1+z)^{2}} O\left(\frac{1}{\sqrt{q}}\right)$. Then we have Theorem 2. Let $\gamma$ be defined as in Lemma 7.

Case $1(1+z)^{2} \gamma \geq 1$.

By Lemma 8 and the fact that $\gamma \geq \frac{1}{(1+z)^{2}}$, we have

$$
\begin{aligned}
P(z \leq \widehat{Y} \leq w) \leq P(z \leq \widehat{Y}) & =P(1+z \leq 1+\widehat{Y}) \\
& \leq \frac{E|\widehat{Y}+1|^{4}}{(1+z)^{4}} \\
& \leq C \frac{E|\widehat{Y}|^{4}}{(1+z)^{4}}+\frac{C}{(1+z)^{4}} \\
& \leq \frac{C}{(1+z)^{4}} \\
& \leq \frac{C \gamma}{(1+z)^{2}} .
\end{aligned}
$$

By Hölder's inequality and Lemma 3(1), E| $\widetilde{Y}-\left.\widehat{Y}\right|^{3}=O\left(\frac{1}{q \sqrt{q}}\right)$. Then $\gamma=O\left(\frac{1}{\sqrt{q}}\right)$ and we have $P(z \leq W \leq w) \leq$ $\frac{1}{(1+z)^{2}} O\left(\frac{1}{\sqrt{q}}\right)$.

Case $2(1+z)^{2} \gamma<1$.

Let $f: \mathbb{R} \rightarrow \mathbb{R}$ be defined by

$$
f(t)= \begin{cases}0 & \text { if } \quad t<z-\gamma \\ (1+t+\gamma)^{3}(t-z+\gamma) & \text { if } \quad z-\gamma \leq t \leq w+\gamma \\ (1+t+\gamma)^{3}(w-z+2 \gamma) & \text { if } \quad t>w+\gamma\end{cases}
$$

Then $f$ is a non decreasing function satisfying

$$
f^{\prime}(t) \geq \begin{cases}(1+z)^{3} & \text { for } z-\gamma<t<w+\gamma \\ 0 & \text { otherwise }\end{cases}
$$

Since $f$ is a continuous and piecewise continuously differentiable function, by Lemma 4 we have

$$
E \widehat{Y} f(\widehat{Y})=E \int_{-\infty}^{\infty} f^{\prime}(\widehat{Y}+t) K(t) d t+\widetilde{\Delta} f(\widehat{Y})
$$


Let $U_{\gamma}$ be defined as in Lemma 7, we observe that

$$
\begin{aligned}
& E \int_{-\infty}^{\infty} f^{\prime}(\widehat{Y}+t) K(t) d t \\
& \geq(1+z)^{3} E \mathbb{I}(z \leq \widehat{Y} \leq w) \int_{|t| \leq \gamma} K(t) d t \\
& =\frac{(q-1)(1+z)^{3}}{4} E \mathbb{I}(z \leq \widehat{Y} \leq w)|\widehat{Y}-\widehat{Y}| \min (\gamma,|\widehat{Y}-\widetilde{Y}|) \\
& =\frac{(q-1)(1+z)^{3}}{4 q(q-1)} E \mathbb{I}(z \leq \widehat{Y} \leq w) U_{\gamma} \\
& \geq \frac{(1+z)^{3}}{4 q} E \mathbb{I}(z \leq \widehat{Y} \leq w) U_{\gamma} \mathbb{I}\left(U_{\gamma} \geq q\right) \\
& \geq \frac{(1+z)^{3}}{4} E \mathbb{I}(z \leq \widehat{Y} \leq w) \mathbb{I}\left(U_{\gamma} \geq q\right) \\
& =\frac{(1+z)^{3}}{4} E\left\{E \mathbb{I}(z \leq \widehat{Y} \leq w)-\mathbb{I}\left(z \leq \widehat{Y} \leq w, U_{\gamma} \leq q\right)\right\} \\
& \geq \frac{(1+z)^{3}}{4}\left\{P(z \leq \widehat{Y} \leq w)-P\left(U_{\gamma} \leq q\right)\right\} .
\end{aligned}
$$

By this fact, Lemma $7(1,2)$, Lemma 8 and $\gamma=O\left(\frac{1}{\sqrt{q}}\right)$,

$$
\begin{aligned}
P(z \leq \widehat{Y} \leq w) \leq & \frac{4}{(1+z)^{3}} E \widehat{Y} f(\widehat{Y})-\frac{4}{(1+z)^{3}} \widetilde{\Delta} f(\widehat{Y})+P\left(U_{\gamma} \leq q\right) \\
\leq & \frac{4}{(1+z)^{3}}(w-z+2 \gamma) E|\widehat{Y}||1+\gamma+\widehat{Y}|^{3} \\
& +\frac{4}{(1+z)^{3}}|\widetilde{\Delta} f(\widehat{Y})|+P\left(E U_{\gamma}-U_{\gamma} \geq 3 q+O(1)-q\right) \\
\leq & \frac{C}{(1+z)^{3}}(w-z+2 \gamma) E|\widehat{Y}|\left|(1+\gamma)^{3}+\widehat{Y}^{3}\right| \\
& +\frac{4}{(1+z)^{3}}|\widetilde{\Delta} f(\widehat{Y})|+P\left(E U_{\gamma}-U_{\gamma} \geq q\right) \\
\leq & \frac{C}{(1+z)^{3}}(w-z+2 \gamma)\left\{E|\widehat{Y}|+E|\widehat{Y}|^{4}\right\} \\
\leq & \frac{C}{(1+z)^{3}}(w-z)+\frac{4}{(1+z)^{3}}|\widetilde{\Delta} f(\widehat{Y})|+\frac{1}{(1+z)^{2}} O\left(\frac{1}{\sqrt{q}}\right) . \\
& +\frac{C}{(1+z)^{3}}|\widetilde{\Delta} f(\widehat{Y})|+\frac{C}{q^{2}} E\left(U_{\gamma}-E U_{\gamma}\right)^{2} \\
& (w-z)+\frac{4}{(1+z)^{3}}|\widetilde{\Delta} f(\widehat{Y})|+\frac{C}{q^{2}} \gamma^{2} O\left(q^{2}\right) \\
& \\
& \\
& \\
&
\end{aligned}
$$


From, Lemma $6(1,2)$, Lemma 8 and the fact that $\gamma=O\left(\frac{1}{\sqrt{q}}\right)$,

$$
\begin{aligned}
& \widetilde{\Delta} f(\widehat{Y})|=| \frac{1}{q} E f(\widehat{Y}) \sum_{i=0}^{q-1} \sum_{j=0}^{q-1} \sum_{k=0}^{q-1} \widehat{Y}_{z}(i, j, k) \mid \\
& \leq \frac{1}{q}(w-z+2 \gamma) E\left|(1+\gamma+\widehat{Y})^{3} \sum_{i=0}^{q-1} \sum_{j=0}^{q-1} \sum_{k=0}^{q-1} \widehat{Y}_{z}(i, j, k)\right| \\
& \leq \frac{C}{q}(w-z+2 \gamma) E\left|(1+\gamma)^{3} \sum_{i=0}^{q-1} \sum_{j=0}^{q-1} \sum_{k=0}^{q-1} \widehat{Y}_{z}(i, j, k)\right| \\
& +\frac{C}{q}(w-z+2 \gamma) E\left|\widehat{Y}^{3} \sum_{i=0}^{q-1} \sum_{j=0}^{q-1} \sum_{k=0}^{q-1} \widehat{Y}_{z}(i, j, k)\right| \\
& \leq C(w-z+2 \gamma)\left(1+\gamma^{3}\right) \frac{1}{q} \sum_{k=0}^{q-1} E\left|\sum_{i=0}^{q-1} \sum_{j=0}^{q-1} \widehat{Y}_{z}(i, j, k)\right| \\
& +\frac{C}{q}(w-z+2 \gamma) E\left|\widehat{Y}^{3}\left(\sum_{i=0}^{q-1} \sum_{j=0}^{q-1} \sum_{k=0}^{q-1} Y(i, j, k)-\sum_{i=0}^{q-1} \sum_{j=0}^{q-1} \sum_{k=0}^{q-1} Y_{z}(i, j, k)\right)\right| \\
& \leq C(w-z+2 \gamma)\left(1+\gamma^{3}\right) E\left|\sum_{i=0}^{q-1} \sum_{j=0}^{q-1} \widehat{Y}_{z}\left(i, j, \rho_{\pi}(i, j)\right)\right| \\
& +\frac{C}{q}(w-z+2 \gamma) E\left|\widehat{Y}^{3} \sum_{i=0}^{q-1} \sum_{j=0}^{q-1} \sum_{k=0}^{q-1} Y(i, j, k)\right| \\
& +\frac{C}{q}(w-z+2 \gamma) E\left|\widehat{Y}^{3} \sum_{i=0}^{q-1} \sum_{j=0}^{q-1} \sum_{k=0}^{q-1} Y_{z}(i, j, k)\right| \\
& \leq C(w-z+2 \gamma)\left(1+\gamma^{3}\right) E|\widehat{Y}| \\
& +\frac{C}{q}(w-z+2 \gamma)\left\{E \widehat{Y}^{4}\right\}^{\frac{3}{4}}\left\{E\left(\sum_{i=0}^{q-1} \sum_{j=0}^{q-1} \sum_{k=0}^{q-1} Y(i, j, k)\right)^{4}\right\}^{\frac{1}{4}} \\
& +\frac{C}{q}(w-z+2 \gamma)\left\{E \widehat{Y}^{4}\right\}^{\frac{3}{4}}\left\{E\left(\sum_{i=0}^{q-1} \sum_{j=0}^{q-1} \sum_{k=0}^{q-1} Y_{z}(i, j, k)\right)^{4}\right\}^{\frac{1}{4}} \\
& \leq C(w-z+2 \gamma)\left(1+\gamma^{3}\right)+\frac{C}{q}(w-z+2 \gamma) O\left(q^{\frac{1}{2}}\right) \\
& +\frac{C}{q}(w-z+2 \gamma) \frac{1}{(1+z)^{\frac{1}{2}}} O\left(q^{-\frac{1}{2}}\right) \\
& \leq C(w-z+2 \gamma)\left(1+O\left(\frac{1}{\sqrt{q}}\right)\right) \\
& \leq C(w-z)+O\left(\frac{1}{\sqrt{q}}\right) \text {. }
\end{aligned}
$$

From this fact and (1), we can conclude that

$$
P(z \leq \widehat{Y} \leq w) \leq \frac{C}{(1+z)^{3}}(w-z)+\frac{1}{(1+z)^{2}} O\left(\frac{1}{\sqrt{q}}\right) .
$$

\section{Acknowledgment}

The authors would like to thank Prof. Kritsana Neammanee who give an idea for improving the boundedness.

\section{References}

Davis, J., Huynh, T. V., Kessler, A., Oravec, J. \& Wark, S. (2007). Orthogonal array experiment for architecting a system of systems responding to small boat attacks. Systems Engineering, 10, Issue 3, 241-259.

Davis, P. J. \& Rabinowitz, P. (1984). Methods of Numerical Integration. (2nd ed.). Orlando FL: Academic Press. 
Evans, M. \& Swartz, T. (2000). Approximating Integrals via Monte-Carlo and Deterministic Methods. Oxford Univ, Press.

Laipaporn, K. \& Neammanee, K. (2006). A non-uniform concentration inequality for randomized orthogonal array sampling design. Thai Journal of Mathematics., 4, 11-34.

Laipaporn, K. \& Neammanee, K. (2007). A non-uniform bound on Normal Approximation of Randomized Orthogonal Array Sampling Designs. Int. Math Forum., 2, 48, 2847-2367.

Laipaporn, K. \& Neammanee, K. (2008). A uniform bound on a combinatorial central limit theorem for randomize orthogonal array sampling design. Stochastic Analysis and Application., 26, 243-255.

Laipaporn, K., Neammanee, K. \& Sungkamong, K. (2009). An Improvement of Normal Approximation of Randomized Orthogonal Array Sampling Designs. Stochastic Analysis and Application., 27, 713-720.

Lazic, L. \& Mastorakis, N. (2008). Orthogonal array application for optimal combination of software defect detection techniques choices. WSEAS Transactions On Computers, 7, Issue 8, 1319-1336.

Lee, K. H., Park, G. J., Park, J. S. \& Yi, J. W. (2003). An optimization algorithm using orthogonal arrays in discrete design space. Finite Elements in Analysis and Design, 40, Issue 1, 121-135.

Lin, K. P., Shih, Y. C. \& Yang, G. K. (2008). Applying orthogonal arrays method to solve fuzzy interval for fuzzy weighted average. The 3rd International Conference on Innovative Computing Information and Control, 42, 18-20.

Loh, W. L. (1996). A combinatorial central limit theorem for randomized orthogonal array sampling designs. Ann. Statist., 24, 1209-1224.

Owen, A. B. (1992a). A central limit theorem for Latin Hypercube sampling. J. R. Statist. Soc. Ser.B, 54, $541-551$.

Owen, A. B. (1992b). Orthogonal array for computer experiments, integration and visualization. Statist. Sinica., 2, 439-452.

Owen, A. B. (1997). Monte-Carlo variance of scrambled net quadrature. SIAM J. Numer. Anal., 34, 1884-1910.

Patterson, H. D. (1954). The errors of lattice sampling. J. Roy. Statist. Soc. Ser. B 16, 140-149.

Tang, B. (1993). Orthogonal array-based Latin hypercubes. J. Amer. Statist. Assoc., 88, 1392-1397. 